\title{
Regular Bardeen Black Holes in Anti-de Sitter Spacetime versus Kerr Black Holes through Particle Dynamics
}

\author{
Bakhtiyor Narzilloev 1,2,3, Javlon Rayimbaev 1,2,3,4,5, Ahmadjon Abdujabbarov 1,3,4,6,7 \\ and Bobomurat Ahmedov 1,3,7,*(D) \\ 1 Ulugh Beg Astronomical Institute, Astronomy Street 33, Tashkent 100052, Uzbekistan; \\ nbakhtiyor18@fudan.edu.cn (B.N.); javlon@astrin.uz (J.R.); ahmadjon@astrin.uz (A.A.) \\ 2 College of Engineering, Akfa University, Kichik Halqa Yuli Street 17, Tashkent 100095, Uzbekistan \\ 3 Faculty of Physics, National University of Uzbekistan, Tashkent 100174, Uzbekistan \\ 4 Institute of Nuclear Physics, Ulugbek 1, Tashkent 100214, Uzbekistan \\ 5 Power Engineering Faculty, Tashkent State Technical University, Tashkent 100095, Uzbekistan \\ 6 Shanghai Astronomical Observatory, 80 Nandan Road, Shanghai 200030, China \\ 7 Department of Physics Tashkent Institute of Irrigation and Agricultural Mechanization Engineers, \\ Kori Niyoziy, 39, Tashkent 100000, Uzbekistan \\ * Correspondence: ahmedov@astrin.uz
}

Citation: Narzilloev, B.

Rayimbaev, J.; Abdujabbarov, A.; Ahmedov, B. Regular Bardeen Black Holes in Anti-de Sitter Spacetime versus Kerr Black Holes through Particle Dynamics. Galaxies 2021, 9 , 63. https://doi.org/10.3390/ galaxies 9030063

Academic Editor: Saurya Das

Received: 31 July 2021

Accepted: 2 September 2021

Published: 6 September 2021

Publisher's Note: MDPI stays neutral with regard to jurisdictional claims in published maps and institutional affiliations.

Copyright: (c) 2021 by the authors. Licensee MDPI, Basel, Switzerland. This article is an open access article distributed under the terms and conditions of the Creative Commons Attribution (CC BY) license (https:// creativecommons.org/licenses/by/ $4.0 /)$.
Abstract: In this work, test particle dynamics around a static regular Bardeen black hole (BH) in Anti-de Sitter spacetime has been studied. It has been shown for neutral test particles that parameters of a regular Bardeen black hole in Anti-de Sitter spacetime can mimic the rotation parameter of the Kerr metric up to the value $a \approx 0.9$ providing the same innermost stable circular orbit (ISCO) radius. We have also explored the dynamics of magnetized particles with a magnetic dipole moment around a magnetically charged regular Bardeen black hole in Anti-de Sitter spacetime. As a realistic astrophysical scenario of the study, we have treated neutron stars orbiting a supermassive black hole (SMBH), in particular, the magnetar PSR J1745-2900 orbiting Sgr $\mathrm{A}^{*}$ with the parameter $\beta=10.2$, as magnetized test particles. The magnetized particles dynamics shows that the parameter $\beta$, negative values of cosmological constant and magnetic charge parameter of the central $\mathrm{BH}$ cause a decrease in the ISCO radius. We have compared the effects of the magnetic charge of the Bardeen $\mathrm{BH}$ with the spin of rotating Kerr $\mathrm{BH}$ and shown that magnetic charge parameter can mimic the spin in the range $a / M \simeq(0,0.7896)$ when $\Lambda=0$ at the range of its values $g / M \simeq(0,0.648)$.

Keywords: einstein-gauss-bonnet gravity; magnetized particles; magnetic field; center-of-mass energy

PACS: 04.50.-h; 04.40.Dg; 97.60.Gb

\section{Introduction}

The curvature singularity at the origin of compact objects is formed as a result of a gravitational collapse of the end state of the evolution of massive stars. It is the one of the main fundamental problems of the general relativity being the classical theory. One of the first attempts to solve this issue has been proposed by Bardeen [1]. In their pioneering work, it was suggested that the regular black hole solution describing the nonsingular compact object surrounded with the event horizon satisfies the weak energy conditions. The solution obtained by Bardeen is not a vacuum solution of the Einstein equations, and its physical interpretation has been delayed for decades. The physical interpretation of this type of solutions has been proposed in the re-obtained solution of regular black hole by AyonBeato and Garcia [2-5]. They have obtained the regular black hole solution introducing the nonlinear magnetic monopole charge of the black hole, which affects the gravitational field of the latter. This is a new, charged black hole solution of the Einstein field equations coupled with nonlinear electrodynamics. The thermodynamic properties of Bardeen's solution have been investigated in [6]. The properties of the regular black hole solutions 
with nonlinear electric and magnetic charges have been studied in [7-18]. In the framework of tensor-vector-scalar (TeVeS) gravity proposed by Moffat and called Modified Gravity (MOG), the regular black hole solutions have been also discussed [19]. Hayward [20] has also proposed an alternative regular black hole solution that is free of an electric charge. The test particle motion around a regular black hole has been studied in [21-26]. The extension of the study of particle motion to the case of no-horizon highly curved spacetimes, being complementary to the regular black hole spacetimes, has been described in [27]. The motion of test particles around a no-horizon regular black hole is similar to one around the naked singularity Reissner-Nordström [28-31] or Kehagias-Sfetsos spacetimes [32-34]. However, there are fundamental differences observed in geodesic motion around the Kerr naked singularity [35-37]. Deformed spacetime properties have been studied in Refs. [38-47]. The prospects of cosmological dark energy for a dynamical theory have been discussed in [48], and the cosmological transitions with a change in metric signature have been investigated in [49]. Bardeen black hole spacetime is not a solution of Einstein field equations. However, regular black hole solutions being the solutions of Einstein field equations also exist in the literature, see, e.g., the non-commutative black holes considered in [50,51].

Einstein's theory of gravity has been tested in both weak and strong field regimes using Solar system tests and observations of the shadow of a supermassive black hole $[52,53]$ at the center of elliptical galaxy M87. It has also been concluded that the analysis of detected gravitational waves by LIGO-Virgo collaboration $[54,55]$ also justifies the general relativity within the current accuracy of the gravity wave experiments. The GRAVITY experiment has justified the gravitational redshift of S2 star infrared (IR) radiation orbiting Sgr A* in the strong field regime. On the other hand, these experiments allow testing and obtain constraints on modified and/or alternative theories of gravity. One of the possible ways of testing the gravity theory is to analyze the $\mathrm{X}$-ray observations from Active galactic nuclei (AGN), and numerous works have been completed, e.g., in Refs. [56-58]. The Kerr black hole having two parameters, namely the total mass and angular momentum, is the most favorable in relativistic astrophysics. However, a recent study performed has indicated that the test electric charge of the supermassive black hole can mimic the spin of the black hole through the test particle's dynamics. It has been shown that the unscreened charge of supermassive black hole Sgr $\mathrm{A}^{*}$ at the center of our galaxy, estimated as $3 \times 10^{8} \mathrm{C}$, can effectively mimic the spin parameter of a black hole up to 0.6 of its maximal value, which is reflected in a considerable shift of the innermost stable circular orbits for charged particles. Consequently, the models estimating the spin of a black hole may require reconsideration due to arising uncertainty in the measurements of the black hole spin (see, e.g., [59]). These uncertainties motivated us to explore black holes with possible extra parameters beyond the standard ones, and a regular Bardeen black hole with a magnetic charge is the case studied in this work.

The effects of the electromagnetic field surrounding the compact object on electrically and magnetically charged particles are also essential. Astrophysical black hole solutions in general relativity according to the no-hair theorem cannot have their own intrinsic magnetic field [60]. However, a black hole can be considered as immersed in an external asymptotically uniform magnetic field [61], and due to spacetime curvature, the structure of the electromagnetic field will be changed in the black hole close environment. These modifications, at the same time, affect different properties of a black hole [62-67].

We refer the readers to Refs. [67-80] for detailed information about the structure of the spacetime and test particles motion around black holes in the presence of a magnetic field. For more studies on the electromagnetic field structure and particle motion around black holes in different gravity models, see, e.g., [22,81-114].

The magnetized particles motion around non-rotating and rotating black holes in the presence of an external magnetic field have been studied in $[115,116]$. Further developments of the magnetized particles motion around a black hole in different gravity models can be found in [117-130]. 
In this paper, we study the neutral and magnetized particles motion around a Bardeen regular black hole in Anti-de Sitter spacetime. The paper is organized as follows: Test particle motion around Bardeen $\mathrm{BH}$ in Anti-de Sitter spacetime is studied in Section 2. Section 3 is devoted to studying the magnetically charged particle motion around the Bardeen regular black hole in Anti-de Sitter spacetime. The dynamics of the dipolar magnetized particles around the regular black hole in the presence of a cosmological constant is studied in Section 4. We summarize our results in Section 5. We use the system where $G=1=c$, and Greek (Latin) indices run values $0,1,2,3(1,2,3)$.

\section{Test Particle Motion around Bardeen BH in Anti-de Sitter Spacetime}

There are several ways of obtaining the regular black hole solutions using the nonlinear electrodynamics, including the Born-Infield extension of the Maxwell theory [131-134]. On the other hand, the explored Bardeen regular black hole solution in GR coupled with nonlinear electrodynamics has the right limiting cases and better describes astrophysical scenarios compared to the regular black solutions in the Born-Infield gravity theory. For this reason, in this work, we consider the special extension to the nonlinear Electrodynamics and the corresponding regular black hole solution obtained in [135]

$$
\begin{aligned}
d s^{2} & =-f(r) d t^{2}+f(r)^{-1} d r^{2}+r^{2} d \Omega^{2}, \\
f(r) & =1-\frac{2 M r^{2}}{\left(r^{2}+g^{2}\right)^{\frac{3}{2}}}-\frac{\Lambda r^{2}}{3},
\end{aligned}
$$

where $M, g$ and $\Lambda$ are the mass of a central object, magnetic charge parameter and cosmological constant, respectively. One can check the regularity of the spacetime of the central object by calculating the Kretschman scalar that is the square of the Riemann tensor $\mathcal{K}=R_{\alpha \beta \mu \nu} R^{\alpha \beta \mu \nu}$, which has the following expression for the spacetime metric above

$$
\begin{aligned}
& \mathcal{K}=\frac{8 \Lambda^{2}}{3}+\frac{8 \Lambda g^{2} M}{\left(g^{2}+r^{2}\right)^{7 / 2}}\left(4 g^{2}-r^{2}\right) \\
& +\frac{12 M^{2}}{\left(g^{2}+r^{2}\right)^{7}}\left(8 g^{8}-4 g^{6} r^{2}+47 g^{4} r^{4}-12 g^{2} r^{6}+4 r^{8}\right) .
\end{aligned}
$$
$r=0$.

It immediately comes out that the metric is indeed regular everywhere, including

The event horizon of a black hole is characterized by the condition $g^{r r}=0$ or equivalently $f(r)=0$ for this spacetime. From this condition, one can plot the dependence between the event horizon radius and the parameters of the spacetime metric $g$ and $\Lambda$, as shown in Figure 1. From the figure, it is apparent that the increase of the magnetic charge parameter decreases the radius of the event horizon, while the increase of the cosmological constant makes it bigger. Since both parameters can change the event horizon radius, one can assume that these parameters can replace the effect of the spin parameter $a$ of the well-known Kerr spacetime. However, we will come to this later when we start to deal with the unique observable quantity called the innermost stable circular orbits (ISCOs) of the test particles.

The equation of motion of a test particle can be obtained by solving the HamiltonJacobi equation that reads

$$
g^{\alpha \beta} \frac{\partial S}{d x^{\alpha}} \frac{\partial S}{d x^{\beta}}=-m^{2}
$$

where $S$ defines the action for the test particle, and $m$ is its mass. 


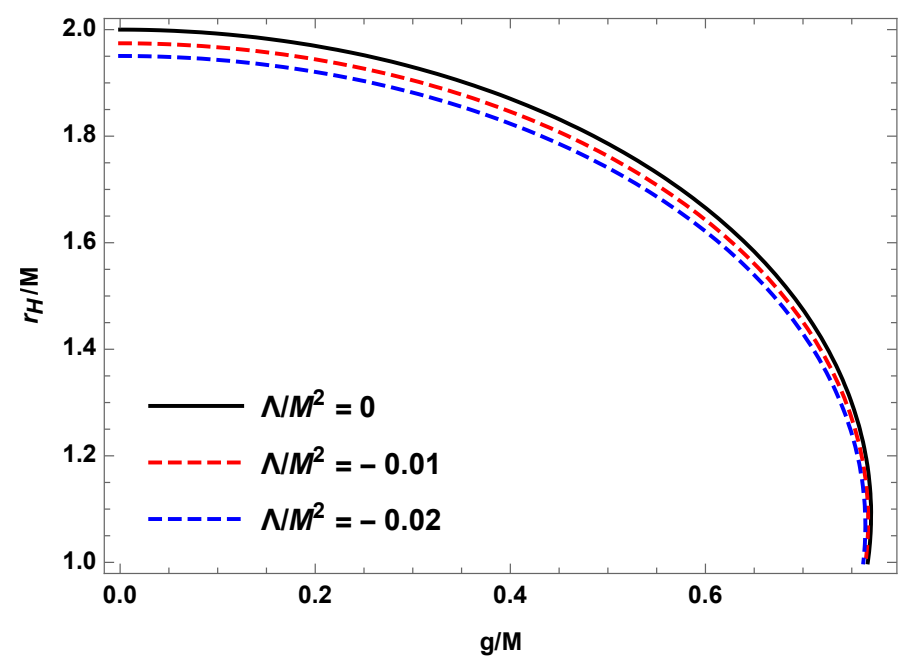

Figure 1. The dependence of the event horizon radius on the parameters $g$ and $\Lambda$.

The action of the test particle moving around the central object can be easily constructed from the symmetrical properties of the spacetime that reads

$$
S=-E t+L \phi+S_{\theta}+S_{r}
$$

with $E$ and $L$ being the energy and angular momentum of the test particle, respectively. Then the equation of motion (3) takes the following form

$$
\begin{aligned}
& -\frac{E^{2}}{1-\frac{2 M r^{2}}{\left(g^{2}+r^{2}\right)^{3 / 2}}-\frac{\Lambda r^{2}}{3}}+\left(1-\frac{2 M r^{2}}{\left(g^{2}+r^{2}\right)^{3 / 2}}-\frac{\Lambda r^{2}}{3}\right)\left(\frac{\partial S}{d r}\right)^{2} \\
& +\frac{L^{2}}{\sin ^{2} \theta r^{2}}+\frac{1}{r^{2}}\left(\frac{\partial S}{d \theta}\right)^{2}=-m^{2} .
\end{aligned}
$$

The trajectory of the test particle can be plotted from the equation above, as shown in Figure 2, for fixed values of the magnetic charge parameter and cosmological constant. One can see that the increase of the magnetic charge and the decrease of the cosmological constant makes the average radius bigger. We can play with these quantities such that the rotation parameter of the spinning Kerr black hole can be replaced by these quantities, which supplies the idea that these parameters can totally or partially mimic the rotation parameter $a$ of the Kerr one.

The effective potential of the test particle can be obtained from the equation of motion and has the following form for the motion at the equatorial plane $(\theta=\pi / 2)$ :

$$
V_{\mathrm{eff}}=\left(1-\frac{2 M r^{2}}{\left(g^{2}+r^{2}\right)^{3 / 2}}-\frac{\Lambda r^{2}}{3}\right)\left(1+\frac{L^{2}}{r^{2}}\right) .
$$

For fixed values of magnetic charge parameter $g$ and cosmological constant $\Lambda$, one can plot the radial dependence for the obtained effective potential as in Figure 3. 

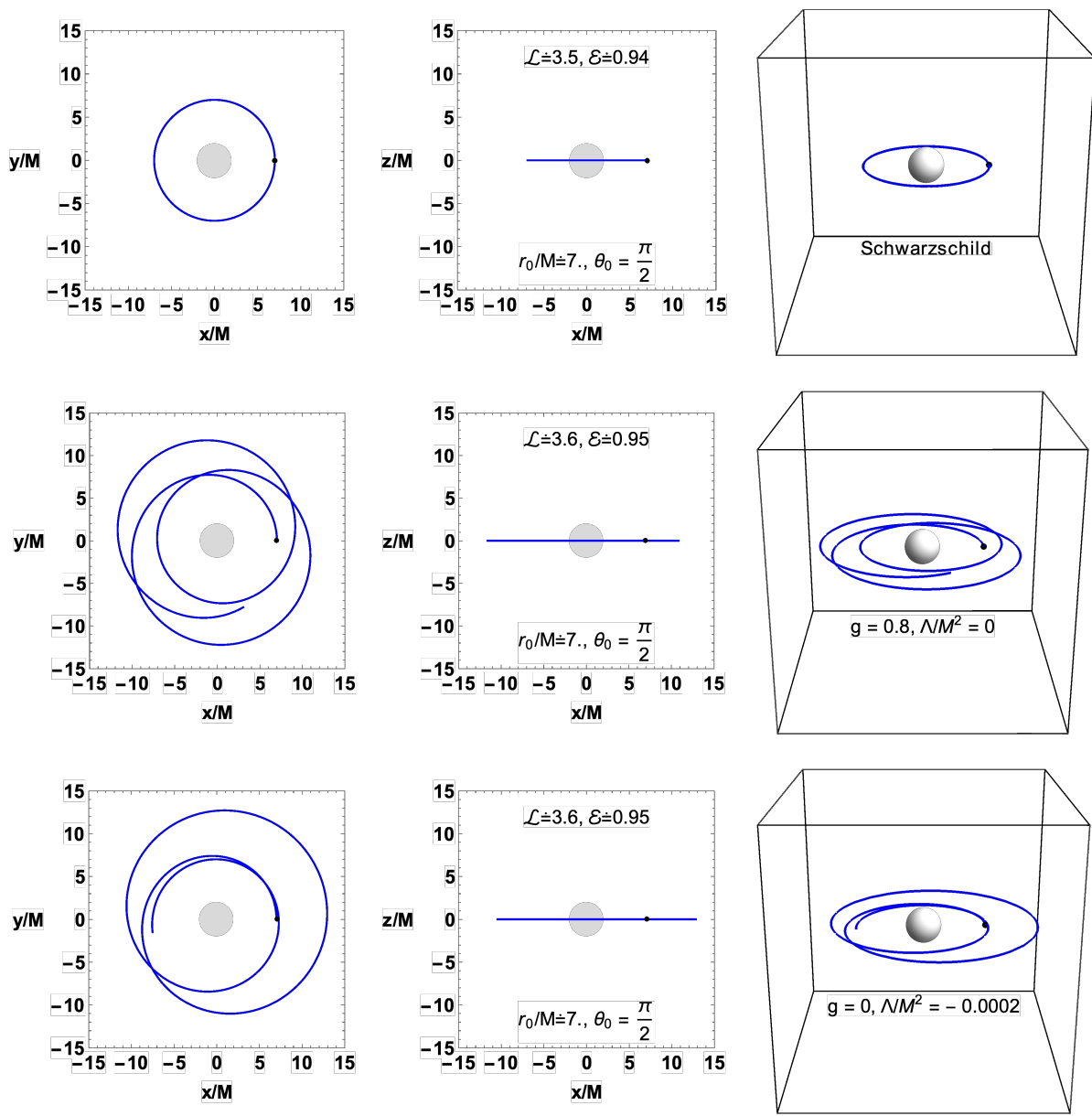

Figure 2. Test particle trajectories for fixed values of $g$ and $\Lambda$. The first line corresponds to the Schwarzschild black hole. The second line corresponds to the magnetically charged black hole. The bottom line is for black hole in Anti-de Sitter spacetime.

$L / m=6, \Lambda / M^{2}=-0.01$



(a)

Figure 3. The radial dependence of the effective potential of a test particle around static regular Bardeen black hole in Anti-de Sitter spacetime. (a) for the fixed cosmological constant and various values of $g$ and (b) for the fixed $g$ and different $\Lambda$.

For circular orbits of the test particle at the equatorial plane, one can set conditions that should be satisfied by the effective potential

$$
V_{\text {eff }}(r)=\mathcal{E}, V_{\text {eff }}^{\prime}(r)=0,
$$

with specific energy, $\mathcal{E}=E / m$. From these conditions, the dependence of the energy from the circular orbit radius of a test particle can be immediately constructed, as presented in 
Figure 4 . One can see that the increase of the magnetic charge parameter decreases the energy near the central object, while the increase of the absolute value of negative cosmological constant makes the energy to increase infinitely since it has an infinite contribution to the spacetime metric as $-\frac{\Lambda r^{2}}{3}$ that increases infinitely for $r \rightarrow \infty$.
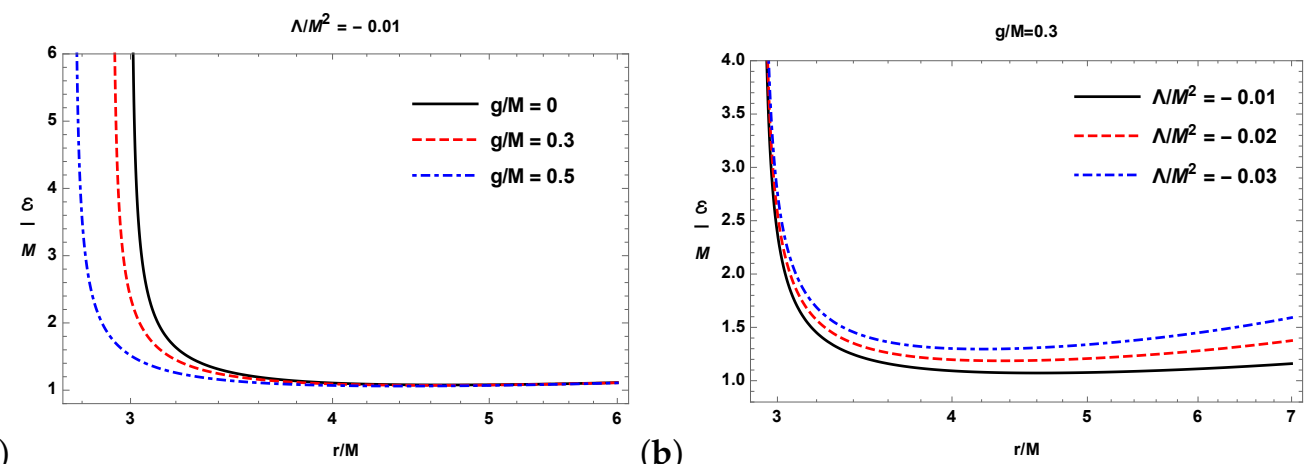

(a)

Figure 4. The dependence of the energy of a test particle on circular orbit radius, static regular Bardeen black hole in Anti-de Sitter spacetime. (a) for a fixed value of $\Lambda$ and different values of $g$. In (b) the value of $g$ is fixed for the different values of $\Lambda$.

Conditions for effective potential can also be used to obtain the radial dependence for angular momentum of the test particle as in Figure 5. It is well known that the minimum value of the angular momentum of the test particle appears at the innermost circular orbits of the test particles and here one can see that the increase of the magnetic charge parameter $g$ reduces this radius, which is similar to the behaviour of the rotation parameter of the Kerr metric. It is also worth noting that for the fixed negative values of cosmological constant, one can see the infinite increase of the angular momentum of the test particle, as in the case for the energy of the test particle also.

(a)


Figure 5. The dependence of the angular momentum of a particle on circular orbit radius static regular Bardeen black hole in Anti-de Sitter spacetime. (a) for the fixed $\Lambda$ and (b) for the fixed $g$.

We have said that the combination of the magnetic charge parameter and cosmological constant can somehow mimic the rotation parameter of the Kerr metric. Now it is time to investigate the possible degeneracy between the rotation parameter and the parameters of regular Bardeen metric in Anti-de Sitter spacetime. The importance of this idea is that it is deeply believed that the black holes in the universe are described by the Kerr metric that defines the rotating gravitational compact object. However, theoretically, it might be possible to have alternate spacetime metric of the black hole that can provide similar behavior of the central massive object with different parameters that could mimic the rotation parameter of the Kerr one. In this work, we focus on the idea that if the black holes in the universe can be described by the regular Bardeen metric in Anti-de Sitter spacetime, then the parameters included in such metric can produce the same ISCO location as if it would be the Kerr black hole. Now our task is to determine how well the rotation 
parameter can be replaced by parameters included in the regular Bardeen metric in Anti-de Sitter spacetime. First, we see the dependence of the ISCO radius from such parameters. To do so, we add an auxiliary condition

$$
V_{\mathrm{eff}}^{\prime \prime}(r)=0
$$

to Equation (7). All together, these conditions provide the sought dependence between the ISCO radius of the test particle orbiting around the regular Bardeen metric in Anti-de Sitter spacetime, as shown in the left panel of Figure 6. It is clearly seen from the left panel that the increase of the magnetic charge parameter in the absence of the cosmological constant can reduce the ISCO radius up to around $3 M$ corresponding to the value $a / M \approx 0.8$ of the rotation parameter of the Kerr spacetime, while the presence of the cosmological constant can "help" to reduce it even better, up to $2 M$, which matches with the value of the rotation parameter $a / M \approx 0.9$.

However, one needs to take into account the fact that the ISCO radius we are using is coordinate-dependent and has no real physical meaning. In order to compare the effects of two different spacetime metrics, one needs to use an invariant quantity, which is coordinate-independent. For this purpose, we introduce the location of ISCO defined as

$$
R_{I S C O}=\frac{l_{\phi}}{2 \pi} .
$$

Here,

$$
l_{\phi}=\int_{0}^{2 \pi} d s_{\phi},
$$

gives us the circumference of the ISCO, and it is an invariant quantity formed from the line element $d s_{\phi}=\left.\sqrt{g_{\phi \phi}}\right|_{r=r_{I S C O}} d \phi$ for the constant $r, \theta=\pi / 2$, and $t$. Now, for the matching values of the location of ISCO, $R_{I S C O}$, of test particles, one can get the desired degeneracy between the spacetime parameters of two different metrics.
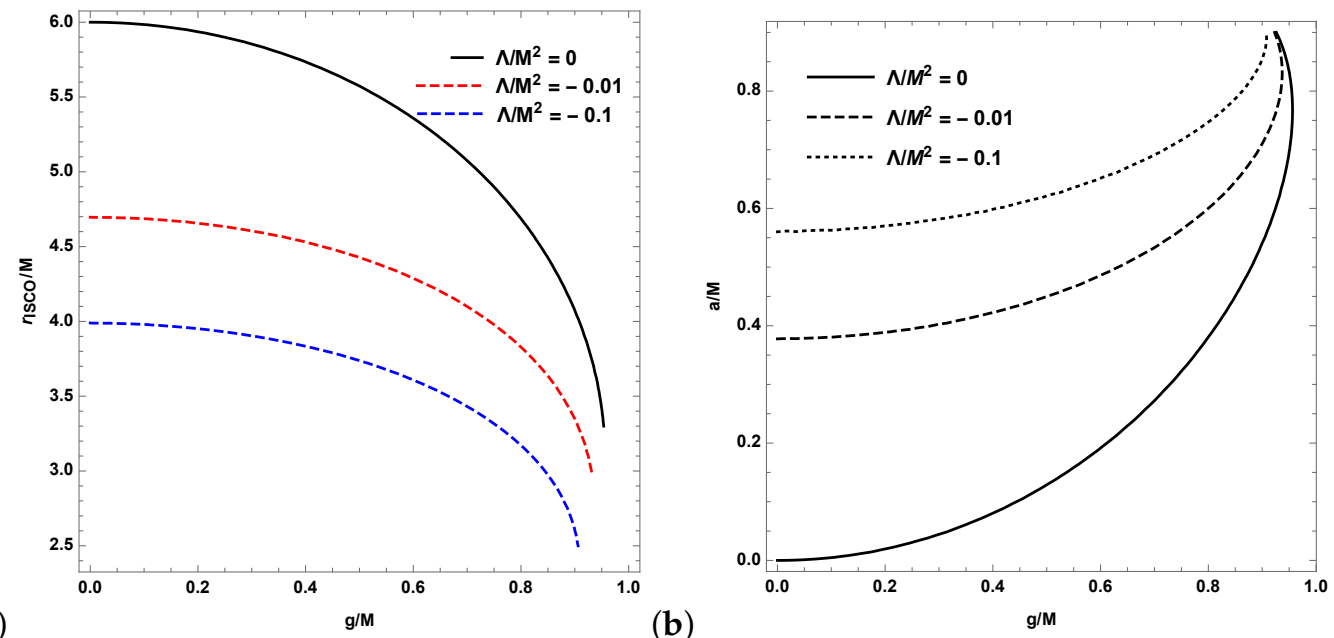

(a)

(b)

Figure 6. The dependence of the ISCO radius from magnetic charge of the black hole and cosmological constant $\Lambda$ (panel (a)), and parameter $a$ from parameters $g$ and $\Lambda$ for the same ISCO location (panel (b)).

The estimated maximum value of the rotation parameter that can be replaced by the combinations of the magnetic charge parameter of the black hole and cosmological constant leads to the exact dependence between these parameters, which provides the same location of ISCO. To do so, one can obtain the degeneracy plot between the parameters of interest, as illustrated in the right panel of Figure 6 for the matching $R_{I S C O}$. From the figure, it is clearly seen that the magnetic charge itself (i.e., with zero cosmological constant) can mimic the rotation parameter up to $a / M \approx 0.8$, which matches with $g / M \approx 1$. One can 
also see that in the combination, these parameters can mimic the rotation parameter up to $a / M \approx 0.9$. Based on the idea that (i) a black hole in the universe can be explained not only as the rotating Kerr one but also as the static regular Bardeen black hole in Anti-de Sitter spacetime, and (ii) based on the same ISCO location, one can conclude that a black hole in the universe with a rotation parameter up to $a / M \approx 0.9$ can be either a Kerr or Bardeen one. It should be mentioned that, when $a=0.9$, the ISCO equation reduces to a regular Bardeen $\mathrm{BH}$, which is found to be in [136].

Figure 7 presents the combination of two parameters $g$ and $\Lambda$ could give the same ISCO location (panel a) or equivalently the same rotation parameter $a$ (panel b).

(a)

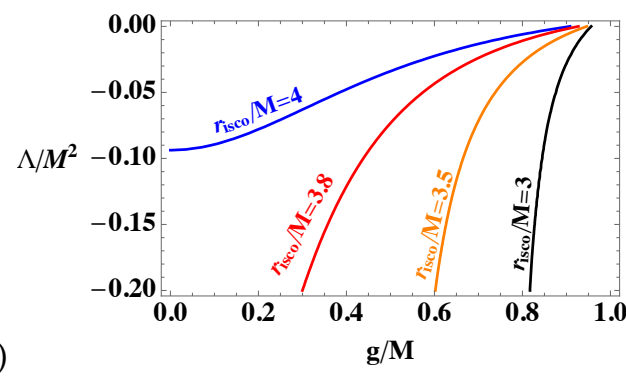

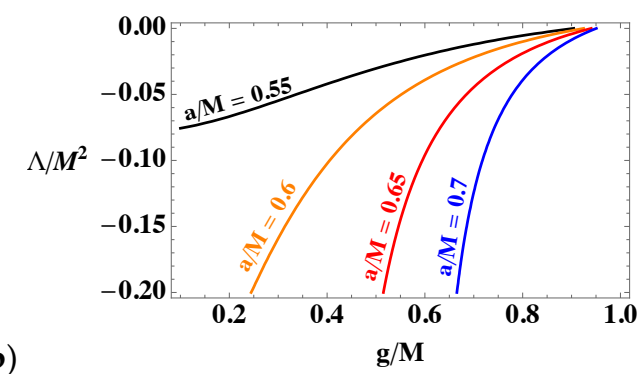

Figure 7. The degeneracy plot between $\Lambda$ and $g$ for the fixed selected values of (a) the ISCO location $r_{\mathrm{ISCO}}$ and $(\mathbf{b})$ the spin parameter $a$.

\section{Magnetically Charged Particle Motion}

Having investigated the neutral particle motion around the regular Bardeen black hole in Anti-de Sitter spacetime, now we turn to the magnetically charged particle motion characterized with the non-zero magnetic monopole. We follow the same idea and corresponding steps as it has been performed in the previous section. Namely, we start from the construction of the equation of motion. For the magnetically charged and electrically neutral particle, the Hamilton-Jacobi equation of motion reads

$$
g^{\alpha \beta}\left(\frac{\partial \mathcal{S}}{\partial x^{\alpha}}+i q_{m} A_{\alpha}^{\star}\right)\left(\frac{\partial \mathcal{S}}{\partial x^{\beta}}+i q_{m} A_{\beta}^{\star}\right)=-m^{2},
$$

where $q_{m}$ is the magnetic charge of the test particle, and four vector $A_{\alpha}^{\star}$ defines the dual vector potential that has the following non-vanishing component (see [80])

$$
A_{t}^{\star}=-\frac{i g}{r} .
$$

The equation of motion then becomes

$$
\begin{aligned}
& -\frac{\left(E-\frac{g q_{m}}{r}\right)^{2}}{1-\frac{2 M r^{2}}{\left(g^{2}+r^{2}\right)^{3 / 2}}-\frac{\Lambda r^{2}}{3}}+\frac{L^{2} \csc \theta}{r^{2}}+\frac{1}{r^{2}}\left(\frac{\partial S}{d \theta}\right)^{2} \\
& +\left(1-\frac{2 M r^{2}}{\left(g^{2}+r^{2}\right)^{3 / 2}}-\frac{\Lambda r^{2}}{3}\right)\left(\frac{\partial S}{d r}\right)^{2}=-m^{2} .
\end{aligned}
$$

The effective potential of the magnetically charged particle moving at the equatorial plane $(\theta=\pi / 2)$ can be written in the following form

$$
V_{\text {eff }}=\frac{q_{m} g}{r}+\sqrt{\left[1+\frac{\mathcal{L}^{2}}{r^{2}}\right]\left[1-\frac{2 M r^{2}}{\left(r^{2}+g^{2}\right)^{\frac{3}{2}}}-\frac{\Lambda r^{2}}{3}\right]},
$$

(with specific angular momentum, $\mathcal{L}=L / m$ ), which recovers the standard Schwarzschild(AdS) effective potential in the case where $g=0$. The radial dependence of this effective 
potential is shown in Figure 8. It is clearly seen from the figures that the additional term in the effective potential due to the electromagnetic interaction between the magnetically charged particle and the field produced by the magnetic charge of the black hole shifts the lines accordingly with the value and sign of the magnetic charge of the test particle.


Figure 8. Effective potential of magnetically charged particle orbiting around regular Bardeen black hole in Anti-de Sitter spacetime.

Equation (7) provides the circular orbit conditions that lead the angular momentum of the magnetically charged test particle to have the radial dependence as plotted in Figure 9. It is seen from the plots that the increase of the magnetic charge of the test particle shifts the minimum of the lines towards the bigger circular orbits. It demonstrates that the increase of such parameter makes the ISCO radius of the test particle smaller. We also see that the shape of the lines does not experience considerable changes compared with the case of a neutral test particle.
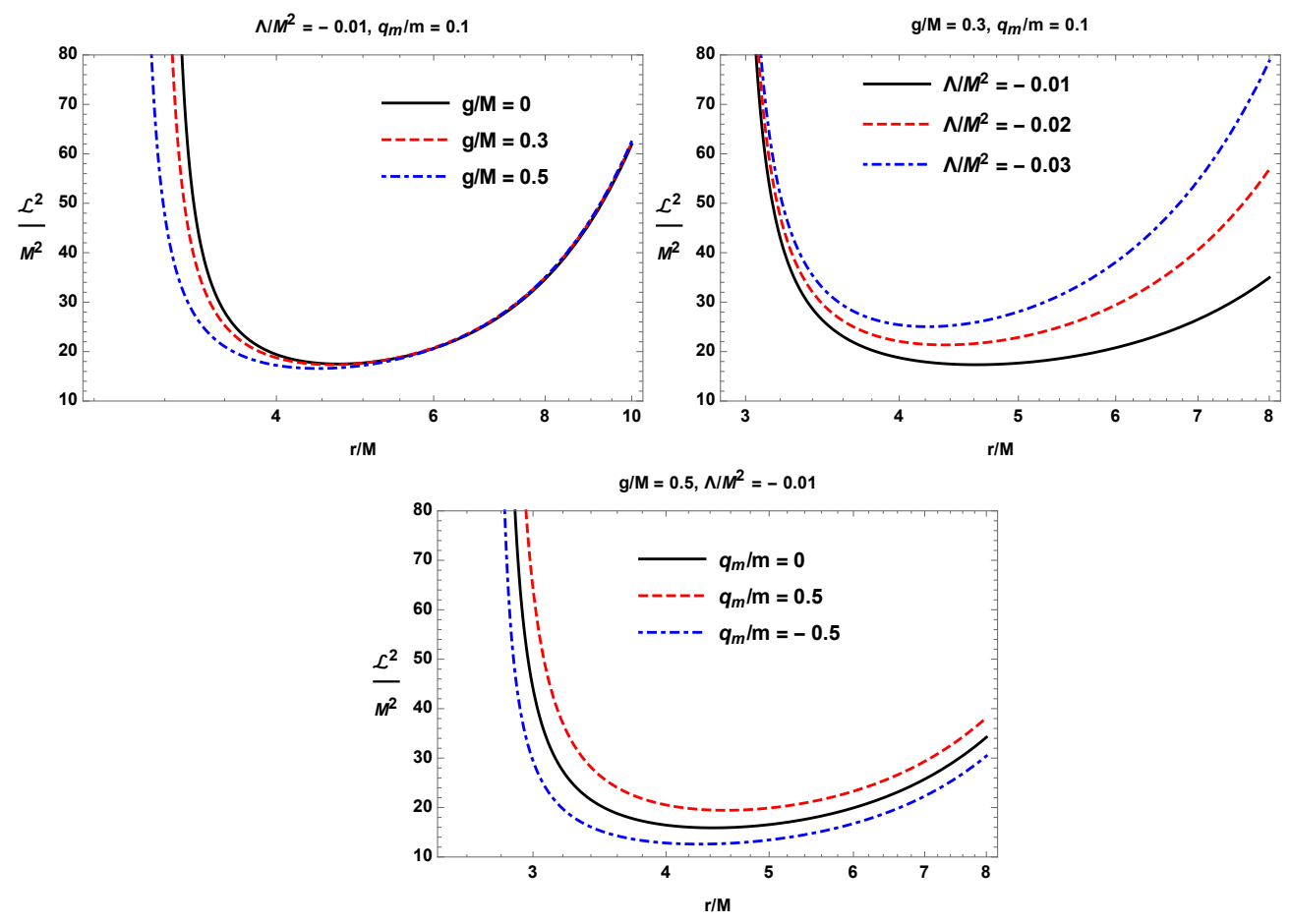

Figure 9. Radial dependence of angular momentum of the magnetically charged particle along circular orbits at the equatorial plane around the regular Bardeen black hole in Anti-de Sitter spacetime.

The idea of this work is based on the investigation of the ISCO radius of the magnetically charged particle moving around the regular Bardeen black hole in Anti-de Sitter spacetime. From the additional condition in Equation (8), one can obtain the aimed relation 
between the ISCO radius and the parameters of the spacetime metric, together with the magnetic charge of the test particle, as plotted in Figure 10. In the given range of the magnetic charge parameter of the test particle, one can see the almost radial dependence between this parameter and the ISCO radius. One can also find that the lines in the figures do not have considerable differences in shape compared to the neutral particle motion that comes from the fact that the interaction between the magnetic field and the magnetically charged test particle is much weaker than the gravitational force of the gravitating object.
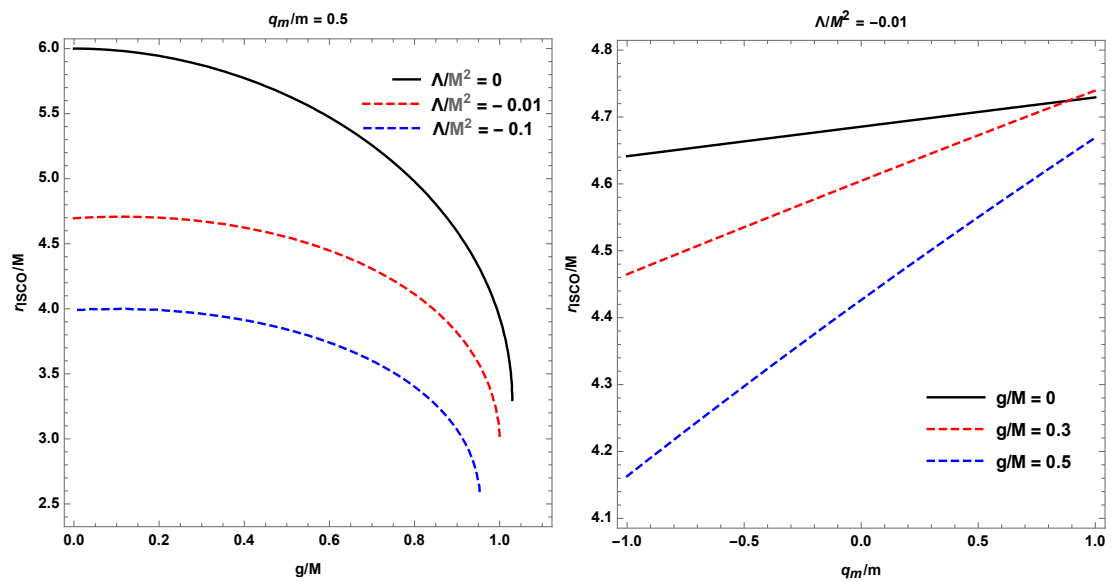

Figure 10. The correspondence between the ISCO radius of the magnetically charged test particle and parameters $g, \Lambda$, and $q_{m}$.

Now we turn to the final part of the section, investigating how the extra interaction due to the influence of the additional parameters embedded in the spacetime metric together with the interaction between magnetically charged test particle and magnetic field around the central black hole can mimic the role of the rotation parameter of the Kerr black hole. Based on the same idea as in the previous section, one can get the degeneracy plots between the rotation parameter $a$ of the Kerr black hole and the parameters $g, \Lambda$, and $q_{m}$ for the orbiting magnetically charged test particle moving around the regular Bardeen black hole in Anti-de Sitter spacetime as plotted in Figure 11. We see that the additional force due to the electromagnetic interaction between the black hole and the magnetically charged test particle does not "help" the metric parameters mimic the rotation parameter of the Kerr black hole.
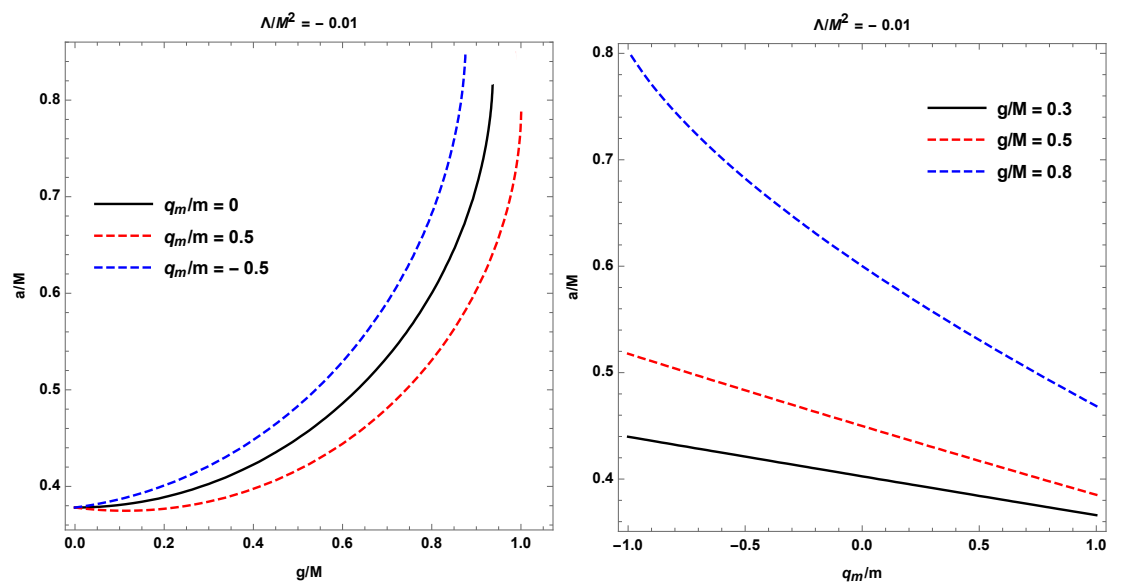

Figure 11. The degeneracy plots between the rotation parameter $a$ and parameters $g, \Lambda$, and $q_{m}$ giving the same ISCO location for the magnetically charged test particle orbiting the regular black hole. 


\section{Dynamics of Dipolar Magnetized Particles in Regular Black Hole Vicinity}

By solving the vacuum Maxwell equations for the electromagnetic field around the central object, one could find the potential of the electromagnetic field as

$$
A_{\alpha}=-\delta_{\alpha}^{\phi} g \cos \theta
$$

where $\delta_{\alpha}^{\phi}$ is Kronecker delta. The non-zero component of the electromagnetic field tensor $F_{\mu \nu}$ can be written through the electromagnetic potential (13), in the following form

$$
F_{\theta \phi}=-g \sin \theta .
$$

One may express the magnetic field of a magnetically charged Bardeen black hole using by the well-known relation

$$
B^{\alpha}=\frac{1}{2} \eta^{\alpha \beta \sigma \mu} F_{\beta \sigma} w_{\mu}
$$

where $w_{\mu}$ is the four velocity of the observer, and $\eta_{\alpha \beta \sigma \gamma}$ is the pseudo-tensorial form of the Levi-Civita symbol $\epsilon_{\alpha \beta \sigma \gamma}$ defined as

$$
\eta_{\alpha \beta \sigma \gamma}=\sqrt{-g} \epsilon_{\alpha \beta \sigma \gamma}, \quad \eta^{\alpha \beta \sigma \gamma}=-\frac{1}{\sqrt{-g}} \epsilon^{\alpha \beta \sigma \gamma},
$$

with $g=\operatorname{det}\left|g_{\mu \nu}\right|=-r^{4} \sin ^{2} \theta$ for the spacetime metric (1) and

$$
\epsilon_{\alpha \beta \sigma \gamma}=\left\{\begin{array}{c}
+1, \text { for even replacements } \\
-1, \text { for odd replacements } \\
0, \text { for the other combinations }
\end{array}\right.
$$

Finally, the orthonormal radial component of the magnetic field generated by the magnetic charge of regular Bardeen BH can be expressed using four-potential (Equation (13)) as

$$
B^{\hat{r}}=\frac{g}{r^{2}} .
$$

The radial component of the magnetic field around magnetically charged black holes formally coincides with the standard Newtonian expression.

\subsection{Equation of Motion}

One may investigate the motion of a magnetized particle with the magnetic dipole moment around a magnetically charged black hole using the following Hamilton-Jacobi (H-J) Equation (see, for example, [115,128])

$$
g^{\mu \nu} \frac{\partial \mathcal{S}}{\partial x^{\mu}} \frac{\partial \mathcal{S}}{\partial x^{v}}=-\left(m-\frac{1}{2} \mathcal{D}^{\mu v} F_{\mu v}\right)^{2},
$$

where the term $\mathcal{D}^{\mu v} F_{\mu \nu}$ is responsible for the interaction between the magnetic field generated by the magnetic charge of the regular Bardeen black hole and magnetic dipole moment $\mu^{v}$ of the magnetized particles.

Let the magnetized particles have a polarization tensor $\mathcal{D}^{\alpha \beta}$, which satisfies the following condition,

$$
\mathcal{D}^{\alpha \beta}=\eta^{\alpha \beta \sigma v} u_{\sigma} \mu_{v}, \quad \mathcal{D}^{\alpha \beta} u_{\beta}=0
$$


One can determine the interaction term $\mathcal{D}^{\mu v} \mathcal{F}_{\mu v}$ using the following relation between the electromagnetic field tensor $F_{\alpha \beta}$ and components of electric $E_{\alpha}$ and magnetic $B^{\alpha}$ fields

$$
F_{\alpha \beta}=w_{\alpha} E_{\beta}-w_{\beta} E_{\alpha}-\eta_{\alpha \beta \sigma \gamma} w^{\sigma} B^{\gamma} .
$$

One may immediately calculate the interaction part of the H-J Equation (19) using the condition given in Equation (20) and the non-zero components of the electromagnetic field tensor as

$$
\mathcal{D}^{\alpha \beta} F_{\alpha \beta}=2 \mu_{\alpha} B^{\alpha}=2 \mu^{\hat{\alpha}} B_{\hat{\alpha}} .
$$

Moreover, one has to assume that the direction of the magnetic dipole moment of the magnetized particle lies at the equatorial plane and parallel to the magnetic field generated by the magnetic charge of the magnetically charged Bardeen $\mathrm{BH}$ and the orthonormal components of the magnetic dipole of the magnetized particle is $\mu^{\hat{i}}=\left(\mu^{\hat{r}}, 0,0\right)$, and it can provide a stable equilibrium to the magnetic interaction between the magnetized particle and the magnetic field. The interaction term can be expressed using Equations (22) and (14) in the following form,

$$
\mathcal{D}^{\alpha \beta} F_{\alpha \beta}=\frac{2 \mu g}{r^{2}},
$$

where $\mu^{2}=\mu_{\hat{i}} \mu^{\hat{i}}$ is the norm of the magnetic dipole moment of the magnetized particle.

Now it is possible to study dynamics of the magnetized particles around the magnetically charged Bardeen $\mathrm{BH}$ at the equatorial plane, where $\theta=\pi / 2$, with $p_{\theta}=0$, using conserved quantities of motion of the magnetized particles, such as energy $p_{t}=-E$ and angular momentum $p_{\phi}=l$, and the energy of the particle can be obtained using Equations (22), (19) and the action (4) in the following form

$$
\dot{r}^{2}=\mathcal{E}^{2}-V_{\text {eff }}(r ; l, \Lambda, \beta, g),
$$

where the effective potential for radial motion of the magnetized particles around the regular Bardeen black hole in Anti-de Sitter spacetime has the following form

$$
V_{\mathrm{eff}}(r ; l, \Lambda, \beta, g)=\left(1-\frac{2 M r^{2}}{\left(r^{2}+g^{2}\right)^{\frac{3}{2}}}-\frac{\Lambda r^{2}}{3}\right)\left[\left(1-\beta \frac{M g}{r^{2}}\right)^{2}+\frac{\mathcal{L}^{2}}{r^{2}}\right],
$$

where the parameter $\beta=\mu /(m M)$ indicates the parameters of the magnetized particle and central object. Neutron stars with their nonzero magnetic moment $\sim 10^{32} \mathrm{G} \cdot \mathrm{cm}^{3}$ and typical mass of $1.4 M_{\odot}$, together with supermassive black holes with the mass in the range $\left(10^{6}-10^{10}\right) M_{\odot}$, can be treated as the system of a black hole and a test particle with a magnetic dipole. This makes the investigation of motion of magnetic dipoles around a black hole astrophysically relevant. One may estimate the coupling parameter $\beta$, assuming the dipole magnetic moment of the neutron star $\mu=(1 / 2) B R^{3}$ with the surface magnetic field $B$ and radius $R$ as

$$
\beta \simeq 0.128\left(\frac{B}{10^{12} \mathrm{G}}\right)\left(\frac{R}{10^{6} \mathrm{~cm}}\right)\left(\frac{m}{1.4 M_{\odot}}\right)^{-1}\left(\frac{M}{10^{6} M_{\odot}}\right)^{-1},
$$

where $m$ and $M$ are the masses of the neutron star and the central SMBH, respectively. The interaction parameter $\beta$ for the magnetar SGR (PSR) J1745-2900 with the magnetic dipole moment $\mu \simeq 1.6 \times 10^{32} \mathrm{G} \cdot \mathrm{cm}^{3}$ and mass $m \simeq 1.4 M_{\odot}$ orbiting the SMBH Sgr A* $\left(M \simeq 3.8 \times 10^{6} M_{\odot}\right)$ has the following value [137]

$$
\beta=\frac{\mu_{\mathrm{PSR} 1745-2900}}{m_{\mathrm{PSR} J 1745-2900} M_{\mathrm{SgrA} *}} \approx 10.2 .
$$


Since the magnetar PSR J1745-2900 is a single-neutron star close to SMBH Sgr A* observed in reality, we will use the parameter $\beta=10.2$ in our further calculations.

Now, one may calculate the characteristics of circular stable orbits for the magnetized particle around the central magnetically charged Bardeen $\mathrm{BH}$ using the standard conditions as

$$
V_{\text {eff }}^{\prime}=0, V_{\text {eff }}^{\prime \prime} \geq 0 \text {. }
$$

The specific angular momentum and energy of the magnetized particle being responsible for circular orbits can be expressed as

$$
\begin{aligned}
\mathcal{L}^{2} & =\frac{1-\beta \frac{g M}{r^{2}}}{\frac{2}{r^{3}}-\frac{6 M r}{\left(g^{2}+r^{2}\right)^{5 / 2}}}\left\{\left(1-\beta \frac{g M}{r^{2}}\right)\left(\frac{2 M r\left(r^{2}-2 g^{2}\right)}{\left(g^{2}+r^{2}\right)^{5 / 2}}-\frac{2 \Lambda r}{3}\right)\right. \\
& \left.-\beta \frac{4 g M}{3 r^{3}}\left[r^{2}\left(\frac{6 M}{\left(g^{2}+r^{2}\right)^{3 / 2}}+\Lambda\right)-3\right]\right\}, \\
\mathcal{E}^{2} & =\frac{r^{4}-\beta^{2} g^{2} M^{2}}{9 r^{4}\left(g^{6}+3 g^{4} r^{2}-3 M r^{4} \sqrt{g^{2}+r^{2}}+3 g^{2} r^{4}+r^{6}\right)} \\
& \times\left[\left(g^{2}+r^{2}\right)^{3 / 2}\left(\Lambda r^{2}-3\right)+6 M r^{2}\right]^{2} .
\end{aligned}
$$

Figure 12 illustrates the radial dependence of the specific angular momentum of magnetized particles for circular motion around a magnetically charged Bardeen black hole in Anti-de Sitter spacetime for the different values of cosmological constant and the black hole charge. Since the magnetar PSR J1745-2900 is treated as a magnetized particle, the value of the parameter $\beta=10.2$ is fixed. One may see from the figure the increase of the absolute value of the cosmological constant and the specific angular momentum with the distance from the central object. The increase of the $\mathrm{BH}$ charge decreases the minimal radius for circular motion. The distance where the angular momentum has a minimum comes close to the central object with the increase of both the cosmological constant and the magnetic charge parameter of the central object.

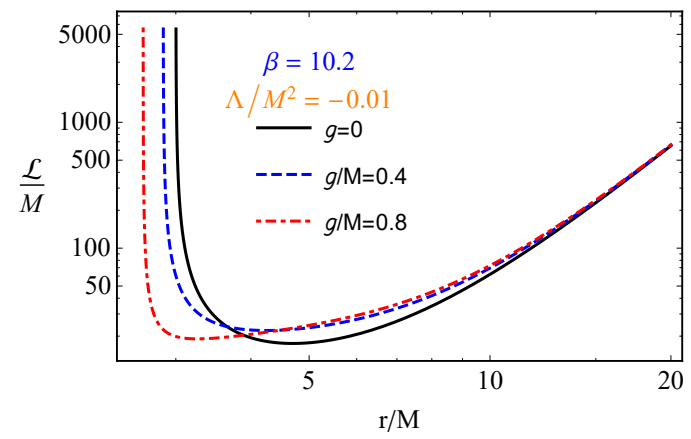

Figure 12. The specific angular momentum of the magnetized particles for circular orbits as a function of the radial coordinate for the different values of magnetic charge parameter $g$ and the parameter $\beta$. In the top panel, the magnetic charge of the $\mathrm{BH}$ is fixed as $g=0.5 M$, and in the bottom panel, the parameter as $\beta=1$.

Figure 13 demonstrates the radial dependence of the specific energy of the magnetized particles moving along circular orbits with the parameter $\beta=10.2$ for the different values of the cosmological constant and magnetic charge of the regular Bardeen black hole. One may see from the figure that the minimum of the energy increases with the increase of the absolute value of the cosmological constant, while with the increase of the magnetic charge, the minimum of the energy decreases. However, the distance where the energy 
is minimum decreases with the increase (decrease) of the magnetic charge of the $\mathrm{BH}$ (cosmological constant).

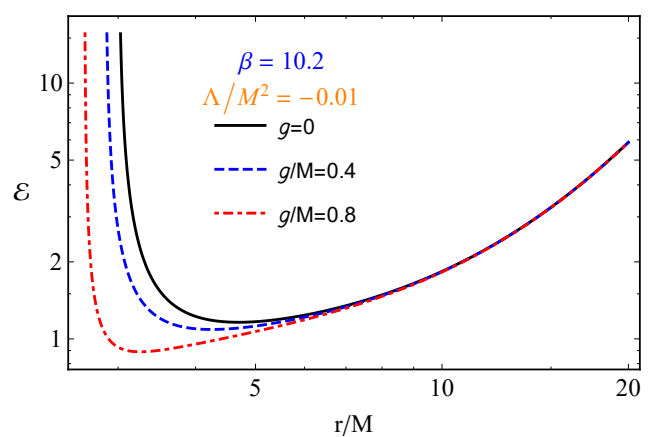

Figure 13. The radial dependence of the specific energy of the magnetized particles around a magnetically charged regular Bardeen black hole in Anti-de Sitter spacetime for circular orbits for the different values of the magnetic charge parameter $g$ and cosmological constant $\Lambda$ for the fixed value of parameter $\beta=10.2$.

\subsection{Stable Circular Orbits}

One can find the equation that allows finding the ISCO radius using the second condition of Equation (28) in the following complicated form.

$$
\begin{aligned}
& 18 \beta^{2} g^{2} M^{4} r^{6}\left(4 g^{2}+5 r^{2}\right)+3 \beta^{2} g^{2} M^{3} r^{4}\left(g^{2}+r^{2}\right)^{3 / 2} \\
& \times\left[4 g^{2}\left(\Lambda r^{2}-9\right)-r^{2}\left(\Lambda r^{2}+21\right)\right]+6 M^{2}\left[2 \beta^{2} g^{2}\left(g^{2}+r^{2}\right)^{5}-3 r^{12}\right] \\
& -4 \Lambda r^{6}\left(g^{2}+r^{2}\right)^{5}+3 M r^{6} \sqrt{g^{2}+r^{2}}\left[g^{2} r^{4}\left(5 \Lambda r^{2}+9\right)+5 \Lambda r^{8}+r^{6}-8 g^{6}\right] \geq 0 .
\end{aligned}
$$

Here we provide analyses, presenting them in plot form.

In order to see the effects of the cosmological parameter and magnetic charge of the regular Bardeen $\mathrm{BH}$, we plot the ISCO radius as a function of the magnetic charge parameter of the BH in Figure 14 for the different values of the cosmological constant and the parameter $\beta$ for the magnetized particle.

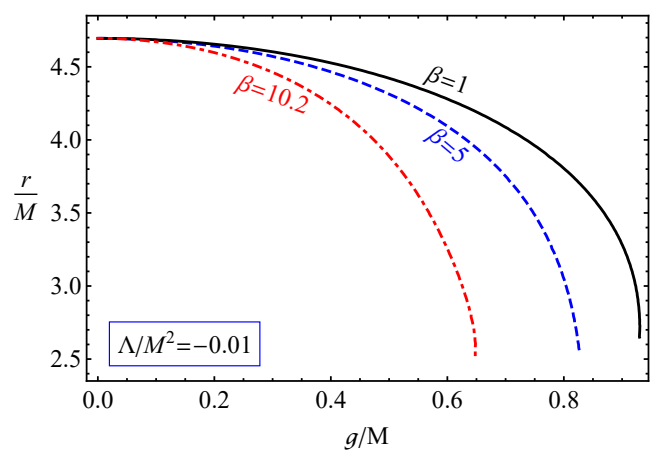

Figure 14. The dependence of the ISCO radius of magnetized particles around the magnetically charged Bardeen black hole in Anti-de Sitter spacetime from the magnetic charge of the black hole for the different values of the parameter $\beta$ (in the top panel) and cosmological constant (in the bottom panel).

One can see from the figure that the increase of the magnetic charge causes a decrease in the ISCO radius, and the ISCO radius increases (decreases) with the increase in the parameter $\beta$ (cosmological constant).

Another interesting and actual problem in relativistic astrophysics is testing different gravity theories in the study of test (neutral, charged and magnetized) particles. The 
spacetime exterior to an astrophysical black hole is described by the Kerr metric, having a non-zero spin parameter $a$. However, the effects of spin of Kerr black holes on the ISCO location of the test particles can be reflected by the parameters of alternative or/and modified theories of gravity, and this may cause the results of analysis to become indistinguishable.

\subsection{Regular Bardeen BH in dS Spacetime versus Kerr BH}

Here, we perform detail analysis for degeneracy values of the spin of Kerr black holes and the magnetic charge of regular Bardeen black holes in Anti-de Sitter spacetime, which may provide the same values of ISCO for magnetized particles by treating the magnetar PSR 1745-2900 orbiting Sgr $A^{*}$ as a magnetized particle with the parameter $\beta=10.2$ for the different values of the cosmological constant.

The ISCO radius of the magnetized particle around a rotating Kerr black hole reads by following the expression in the absence of the external magnetic field (magnetized particle is considered as a neutral test particle) for the corresponding prograde and retrograde orbits as [138]

$$
r_{\text {isco }}=3+Z_{2} \pm \sqrt{\left(3-Z_{1}\right)\left(3+Z_{1}+2 Z_{2}\right)},
$$

where

$$
\begin{aligned}
& Z_{1}=1+(\sqrt[3]{1+a}+\sqrt[3]{1-a}) \sqrt[3]{1-a^{2}} \\
& Z_{2}^{2}=3 a^{2}+Z_{1}^{2}
\end{aligned}
$$

Now, we look for possible ways to distinguish the effects of the magnetic charge of regular Bardeen black hole in Anti-de Sitter spacetime and the rotating Kerr black hole on the dynamics of the magnetized particles with the parameter $\beta=10.2$ for the different values of the cosmological constant.

Figure 15 demonstrates the correspondence between the spin and magnetic charge parameters for the same values of the ISCO location of a magnetized particle with the parameter $\beta=10.2$ for the different values of the cosmological constant. One can see that the magnetic charge of the regular Bardeen BH can mimic the spin of Kerr BH up to $a / M \simeq 0.7896$, with its value in the range of $g / M \in(0,0.648)$ when the cosmological constant is zero. However, as the absolute value of cosmological constant, the mimic range of the rotation parameter decreases. For example, when the cosmological constant $\Lambda=-0.01$ $(\Lambda=-0.05)$, the magnetic charge parameter of the Bardeen BH can mimic the spin parameter of the Kerr BH in the range of $a / M \in(0.3012,0.7896)(a / M \in(0.4236,0.7896))$.



Figure 15. Relations between the magnetic charge of a regular Bardeen black hole in Anti-de Sitter spacetime and the spin of the Kerr black hole, which provides the same value for the ISCO location of the magnetized particle with the parameter $\beta=10.2$ at the different values of the cosmological constant. 


\subsection{Regular Bardeen BH in AdS Spacetime versus Kerr BH for the Matching Radiative Efficiency}

Particles falling into a $\mathrm{BH}$ from the accretion disk around the $\mathrm{BH}$ defines radiation energy released from the accretion disk [139]. The efficiency of the energy release in the accretion disk can be calculated using the expression in the single-particle model,

$$
\eta=1-\mathcal{E}_{\mathrm{ISCO}},
$$

where $\mathcal{E}_{\text {ISCO }}$ corresponds to the specific energy of a particle orbiting at the ISCO.

One can analyze the effect of the magnetic charge of the Bardeen BH in Anti-de Sitter spacetime on the radiative efficiency given by Equation (33).

In Figure 16, we have presented the dependence of the radiative efficiency of the Bardeen $\mathrm{BH}$ in de-Sitter spacetime from the $\mathrm{BH}^{\prime}$ s magnetic charge for the different values of the cosmological constant. One can see from the figure that the efficiency grows with the increase in the charge parameter, up to $40 \%$. It has been also shown in the figure that for smaller values of the cosmological constant, the radiative efficiency also becomes smaller.

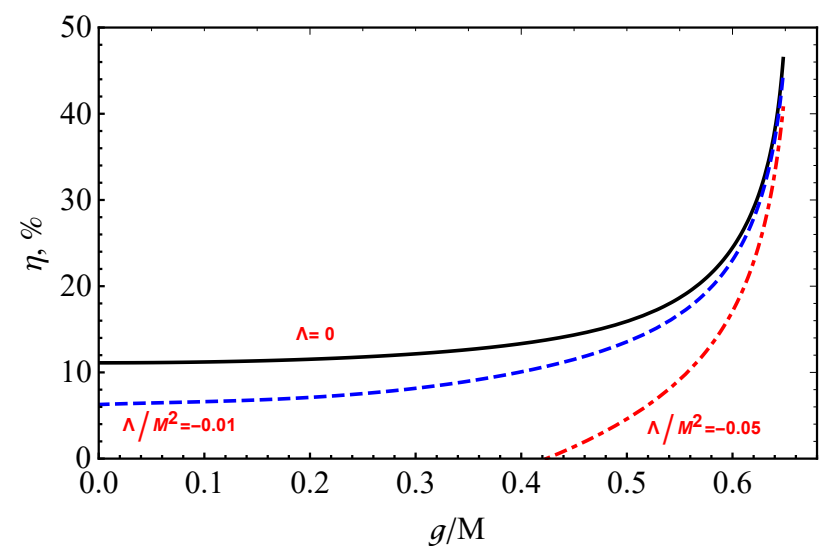

Figure 16. The radiative efficiency of the accretion disk around the regular Bardeen $\mathrm{BH}$ in AdS spacetime as a function of the $\mathrm{BH}$ magnetic charge for the particle with $\beta=10.2$.

One of the ways to test/compare gravity theories using astrophysical observations is connected with the study of the efficiency of the energy release from the accretion disk around a BH. Since the dependence of the radiative efficiency of the Kerr BH from the spin is well known [138], one can now obtain the degeneracy between the spin parameter of the Kerr $\mathrm{BH}$ and the magnetic charge of the Bardeen $\mathrm{BH}$ in AdS spacetime for the matching values of the radiative efficiency, as shown in Figure 17.

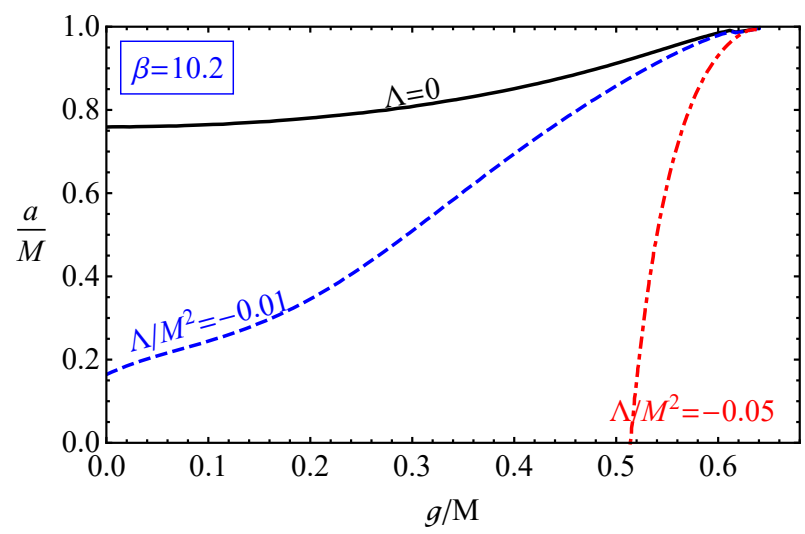

Figure 17. The degeneracy between the spin parameter of the Kerr $\mathrm{BH}$ and the magnetic charge parameter of the Bardeen $\mathrm{BH}$ in AdS spacetime for the matching radiative efficiency. 
One can clearly see from Figure 17 that the range of the mimicking values of the spin parameter increases as the absolute values of the cosmological constant increase and completely mimics the spin when the cosmological constant is $\Lambda=-0.05$. We see that the degeneracy plots presented for the magnetic dipole in Figures 15 and 17 do not recover each other for the given ranges of the parameters. This, in turn, indicates that theoretically, it is possible to distinguish the Bardeen $\mathrm{BH}$ in AdS spacetime from that of the Kerr BH based on the two measurements, namely the measurement of the radius of the inner edge of the accretion disk that matches with the ISCO and the measurement of the radiative efficiency of the accretion disk around a BH.

\section{Conclusions}

This work is devoted to the investigation of the test particle motion around a static regular Bardeen black hole in Anti-de Sitter spacetime. Based on the idea that if a black hole in the universe is believed to be a Bardeen one with a cosmological constant rather than the Kerr one, then the same ISCO location for matching values of the spin parameter of the Kerr metric can be produced by the parameters included in the spacetime metric of interest.

It has been shown for neutral test particles that parameters of a regular Bardeen black hole in Anti-de Sitter spacetime can mimic the rotation parameter of the Kerr metric up to the value $a / M \approx 0.9$ based at the same ISCO location in both cases. It leads to the assumption that black hole candidates assumed to be rotating Kerr ones with a rotation parameter up to this value can also be static ones with a magnetic charge parameter in the presence of a negative cosmological constant.

The similar calculations for the magnetically charged test particles have shown that the additional force due to the electromagnetic interaction influences the results when compared to the neutral ones. Based on the obtained results, it has been justified that this kind of additional interaction does not cause drastic changes in the situation. The black hole characterized by the Bardeen metric in Anti-de Sitter spacetime can mimic the Kerr one with a considerably high rotation parameter $a>0.9$. It shows that the force acting on the test particle is strongly dominated by the spacetime parameters compared to the electromagnetic interaction.

Moreover, we have explored the dynamics of magnetized particles with a non-zero magnetic dipole moment, around a magnetically charged regular Bardeen black hole in Anti-de Sitter spacetime. As a realistic astrophysical scenario of the study, we have treated neutron stars orbiting SMBH, in particular, the magnetar PSR J1745-2900 orbiting Sgr A* with the parameter $\beta=10.2$, as the test magnetized particles. The performed study of the ISCO radius of the magnetized particles has shown that the parameter $\beta$, negative values of cosmological constant and magnetic charge of the central BH causes the ISCO radius to decrease. Finally, as an important issue of relativistic astrophysics, we have compared the effect of the magnetic charge of the Bardeen $\mathrm{BH}$ with one of the spin parameter of rotating Kerr $\mathrm{BHs}$ and shown that the magnetic charge parameter can mimic the spin parameter of Kerr BHs in the range $a / M \simeq(0,0.7896)$ when $\Lambda=0$ is at the range of its values $g / M \simeq(0,0.648)$, while with the increase of the absolute value of cosmological constant, the mimic range of the spin parameter decreases.

The performed study has indicated the possible degeneracy between the parameters of black holes in the dynamics of test particles in a close BH environment. It is concluded that the various $\mathrm{BH}$ parameter extraction methods from observational data have to be applied for correctly probing the nature of the astrophysical black holes.

Author Contributions: Conceptualization, B.N. and J.R.; methodology, A.A.; software, B.N.; validation, B.N., A.A., B.A.; formal analysis, B.N. and J.R.; investigation, B.N. and J.R.; resources, A.A.; data curation, B.A.; writing—original draft preparation, B.N. and J.R.; writing-review and editing, B.A. and A.A.; visualization, B.N. and J.R.; supervision, B.A. and A.A.; project administration, B.A. and A.A. All authors have read and agreed to the published version of the manuscript. 
Funding: Ministry of Innovative Development of the Republic of Uzbekistan: MRB-AN-2019-29, China Scholarship Council: 2018DFH00901.

Institutional Review Board Statement: Not applicable.

Informed Consent Statement: Not applicable.

Data Availability Statement: Not applicable.

Acknowledgments: This research is supported by Grants No. VA-FA-F-2-008, No.MRB-AN-2019-29 of the Uzbekistan Ministry for Innovative Development. J.R. and A.A. thank Silesian University in Opava for the hospitality during their visit. J.R. thanks ERASMUS+ project 608715-EPP-1-2019-1-UZEPPKA2-JP (SPACECOM). B.N. acknowledges support from the China Scholarship Council (CSC), grant No. 2018DFH009013.

Conflicts of Interest: The authors declare no conflict of interest.

\section{References}

1. Bardeen, J. Proceedings of GR5 (Tbilisi, USSR); Dewitt, C., Dewitt, B.S., Eds.; Gordon \& Breach: New York, NY, USA, 1968 ; p. 174.

2. Ayón-Beato, E.; García, A. Regular Black Hole in General Relativity Coupled to Nonlinear Electrodynamics. Phys. Rev. Lett. 1998, 80, 5056-5059. [CrossRef]

3. Ayon-Beato, E. New regular black hole solution from nonlinear electrodynamics. Phys. Lett. B 1999, 464, 25-29. [CrossRef]

4. Ayon-Beato, E.; Garcia, A. Non-Singular Charged Black Hole Solution for Non-Linear Source. Gen. Relativ. Gravit. 1999, $31,629$. [CrossRef]

5. Ayón-Beato, E.; García, A. The Bardeen model as a nonlinear magnetic monopole. Phys. Lett. B 2000, 493, 149-152. [CrossRef]

6. Tzikas, G. Bardeen black hole chemistry. Phys. Lett. B. 2019, 788, 219-224. [CrossRef]

7. Bronnikov, K.A.; Melnikov, V.N.; Shikin, G.N.; Staniukovich, K.P. Scalar, electromagnetic, and gravitational fields interaction: Particlelike solutions. Ann. Phys. 1979, 118, 84-107. [CrossRef]

8. Toshmatov, B.; Stuchlík, Z.; Ahmedov, B.; Malafarina, D. Relaxations of perturbations of spacetimes in general relativity coupled to nonlinear electrodynamics. Phys. Rev. D 2019, 99, 064043. [CrossRef]

9. Toshmatov, B.; Stuchlík, Z.; Schee, J.; Ahmedov, B. Electromagnetic perturbations of black holes in general relativity coupled to nonlinear electrodynamics. Phys. Rev. D 2018, 97, 084058. [CrossRef]

10. Toshmatov, B.; Stuchlík, Z.; Ahmedov, B. Electromagnetic perturbations of black holes in general relativity coupled to nonlinear electrodynamics: Polar perturbations. Phys. Rev. D 2018, 98, 085021. [CrossRef]

11. Toshmatov, B.; Stuchlík, Z.; Ahmedov, B. Comment on "Construction of regular black holes in general relativity". Phys. Rev. D 2018, 98, 028501. [CrossRef]

12. Toshmatov, B.; Bambi, C.; Ahmedov, B.; Stuchlík, Z.; Schee, J. Scalar perturbations of nonsingular nonrotating black holes in conformal gravity. Phys. Rev. D 2017, 96, 064028. [CrossRef]

13. Toshmatov, B.; Bambi, C.; Ahmedov, B.; Abdujabbarov, A.; Stuchlík, Z. Energy conditions of non-singular black hole spacetimes in conformal gravity. Eur. Phys. J. C 2017, 77, 542. [CrossRef]

14. Toshmatov, B.; Stuchlík, Z.; Ahmedov, B. Generic rotating regular black holes in general relativity coupled to nonlinear electrodynamics. Phys. Rev. D 2017, 95, 084037. [CrossRef]

15. Toshmatov, B.; Abdujabbarov, A.; Stuchlík, Z.; Ahmedov, B. Quasinormal modes of test fields around regular black holes. Phys. Rev. D 2015, 91, 083008. [CrossRef]

16. Bronnikov, K.A. Comment on "Regular Black Hole in General Relativity Coupled to Nonlinear Electrodynamics". Phys. Rev. Lett. 2000, 85, 4641. [CrossRef]

17. Bronnikov, K.A. Regular magnetic black holes and monopoles from nonlinear electrodynamics. Phys. Rev. D 2001, 63, 044005. [CrossRef]

18. Dymnikova, I. Regular electrically charged vacuum structures with de Sitter centre in nonlinear electrodynamics coupled to general relativity. Class. Quantum Gravity 2004, 21, 4417-4428. [CrossRef]

19. Moffat, J.W. Black holes in modified gravity (MOG). Eur. Phys. J. C 2015, 75, 175. [CrossRef]

20. Hayward, S.A. Formation and Evaporation of Nonsingular Black Holes. Phys. Rev. Lett. 2006, 96, 031103. [CrossRef] [PubMed]

21. Toshmatov, B.; Abdujabbarov, A.; Ahmedov, B.; Stuchlík, Z. Particle motion and Penrose processes around rotating regular black hole. Astrophys. Space Sci. 2015, 357, 41. [CrossRef]

22. García, A.; Hackmann, E.; Kunz, J.; Lämmerzahl, C.; Macías, A. Motion of test particles in a regular black hole space-time. J. Math. Phys. 2015, 56, 032501. [CrossRef]

23. Patil, M.; Joshi, P.S. Ultrahigh energy particle collisions in a regular spacetime without black holes or naked singularities. Phys. Rev. D 2012, 86, 044040. [CrossRef]

24. Toshmatov, B.; Ahmedov, B.; Abdujabbarov, A.; Stuchlík, Z. Rotating regular black hole solution. Phys. Rev. D. 2014, 89, 104017. [CrossRef] 
25. Rayimbaev, J.; Figueroa, M.; Stuchlík, Z.; Juraev, B. Test particle orbits around regular black holes in general relativity combined with nonlinear electrodynamics. Phys. Rev. D 2020, 101, 104045. [CrossRef]

26. Turimov, B.; Rayimbaev, J.; Abdujabbarov, A.; Ahmedov, B.; Stuchlík, Z.C.V. Test particle motion around a black hole in Einstein-Maxwell-scalar theory. Phys. Rev. D 2020, 102, 064052. [CrossRef]

27. Stuchlík, Z.; Schee, J. Circular geodesic of Bardeen and Ayon-Beato-Garcia regular black-hole and no-horizon spacetimes. Int. J. Mod. Phys. D 2015, 24, 50020. [CrossRef]

28. Pugliese, D.; Quevedo, H.; Ruffini, R. Motion of charged test particles in Reissner-Nordström spacetime. Phys. Rev. D 2011, 83, 104052. [CrossRef]

29. Stuchlík, Z.; Hledik, S. Properties of the Reissner-Nordstrom spacetimes with a nonzero cosmological constant . Acta Phys. Slovaca 2002, 52, 363-407.

30. Pugliese, D.; Quevedo, H.; Ruffini, R. Circular motion of neutral test particles in Reissner-Nordström spacetime. Phys. Rev. D 2011, 83, 024021. [CrossRef]

31. Pugliese, D.; Quevedo, H.; Ruffini, R. Circular motion in Reissner-Nordström spacetime. arXiv 2010, arXiv:1003.2687.

32. Vieira, R.S.S.; Schee, J.; Kluźniak, W.; Stuchlík, Z.; Abramowicz, M. Circular geodesics of naked singularities in the KehagiasSfetsos metric of Hořava's gravity. Phys. Rev. D 2014, 90, 024035. [CrossRef]

33. Stuchlík, Z.; Schee, J. Optical effects related to Keplerian discs orbiting Kehagias-Sfetsos naked singularities. Class. Quantum Gravity 2014, 31, 195013. [CrossRef]

34. Stuchlík, Z.; Slaný, P. Equatorial circular orbits in the Kerr de Sitter spacetimes. Phys. Rev. D 2004, 69, 064001. [CrossRef]

35. Stuchlik, Z. Equatorial circular orbits and the motion of the shell of dust in the field of a rotating naked singularity. Bull. Astron. Institutes Czechoslov. 1980, 31, 129-144.

36. Stuchlík, Z.; Schee, J. Appearance of Keplerian discs orbiting Kerr superspinars. Class. Quantum Gravity 2010, $27,215017$. [CrossRef]

37. Stuchlík, Z.; Schee, J. Ultra-high-energy collisions in the superspinning Kerr geometry. Class. Quantum Gravity 2013, $30,075012$. [CrossRef]

38. Bambi, C. Testing black hole candidates with electromagnetic radiation. Rev. Mod. Phys. 2017, 89, 025001. [CrossRef]

39. Rayimbaev, J.R.; Ahmedov, B.J.; Juraeva, N.B.; Rakhmatov, A.S. Plasma magnetosphere of deformed magnetized neutron star. Astrophys. Space Sci. 2015, 356, 301-308. [CrossRef]

40. Bambi, C.; Barausse, E. Final stages of accretion onto non-Kerr compact objects. Phys. Rev. D 2011, 84, 084034. [CrossRef]

41. Chen, S.; Jing, J. Strong gravitational lensing by a rotating non-Kerr compact object. Phys. Rev. D 2012, 85, 124029. [CrossRef]

42. Narzilloev, B.; Malafarina, D.; Abdujabbarov, A.; Bambi, C. On the properties of a deformed extension of the NUT space-time. Eur. Phys. J. C 2020, 80, 784. [CrossRef]

43. Narzilloev, B.; Malafarina, D.; Abdujabbarov, A.; Ahmedov, B.; Bambi, C. Particle motion around a static axially symmetric wormhole. arXiv 2021, arXiv:2105.09174.

44. Bambi, C.; Caravelli, F.; Modesto, L. Direct imaging rapidly-rotating non-Kerr black holes. Phys. Lett. B 2012, 711, 10-14. [CrossRef]

45. Bambi, C. Testing the space-time geometry around black hole candidates with the analysis of the broad K $\alpha$ iron line. Phys. Rev. D 2013, 87, 023007. [CrossRef]

46. Bambi, C.; Cárdenas-Avendaño, A.; Dauser, T.; García, J.A.; Nampalliwar, S. Testing the Kerr Black Hole Hypothesis Using X-ray Reflection Spectroscopy. Astrophys J. 2017, 842, 76. [CrossRef]

47. Cao, Z.; Nampalliwar, S.; Bambi, C.; Dauser, T.; García, J.A. Testing General Relativity with the Reflection Spectrum of the Supermassive Black Hole in 1H0707-495. Phys. Rev. Lett. 2018, 120, 051101. [CrossRef]

48. Antoniadis, I.; Mazur, O.; Mottola, E. Cosmological dark energy: Prospects for a dynamical theory. New J. Phys. 2007, 9, 11. [CrossRef]

49. Sakharov, A.D. Cosmological Transitions With a Change in Metric Signature. Sov. Phys. JETP 1984, 60, 214-218. [CrossRef]

50. Nicolini, P.; Smailagic, A.; Spallucci, E. Noncommutative geometry inspired Schwarzschild black hole. Phys. Lett. B. 2006, 632, 547-551. [CrossRef]

51. Arraut, I.; Batic, D.; Spallucci, E.; Nowakowski, M. A Non commutative model for a mini black hole. Class. Quantum Gravity 2009, 26, 245006. [CrossRef]

52. Akiyama, K.; Bouman, K.L.; Woody, D.P. First M87 Event Horizon Telescope Results. I. The Shadow of the Supermassive Black Hole. Astrophys. J. 2019, 875, L1. [CrossRef]

53. Akiyama, K.; Alberdi, A.; Alef, W.; Asada, K. First M87 Event Horizon Telescope Results. VI. The Shadow and Mass of the Central Black Hole. Astrophys. J. 2019, 875, L6. [CrossRef]

54. The LIGO Scientific Collaboration.; The Virgo Collaboration. Tests of general relativity with GW150914. arXiv 2016, arXiv:1602.03841.

55. Abbott, B.P.; Abbott, R.; Abbott, T.D.; Abernathy, M.R.; Acernese, F.; Ackley, K.; Adams, C.; Adams, T.; Addesso, P.; Adhikari, R.X.; et al. Observation of Gravitational Waves from a Binary Black Hole Merger. Phys. Rev. Lett. 2016, 116, 061102. [CrossRef]

56. Bambi, C.; Malafarina, D.; Modesto, L. Black supernovae and black holes in non-local gravity. J. High Energy Phys. 2016, $2016,147$. [CrossRef] 
57. Zhou, M.; Cao, Z.; Abdikamalov, A.; Ayzenberg, D.; Bambi, C.; Modesto, L.; Nampalliwar, S. Testing conformal gravity with the supermassive black hole in 1H0707-495. Phys. Rev. D 2018, 98, 024007. [CrossRef]

58. Tripathi, A.; Yan, J.; Yang, Y.; Yan, Y.; Garnham, M.; Yao, Y.; Li, S.; Ding, Z.; Abdikamalov, A.B.; Ayzenberg, D.; et al. Constraints on the spacetime metric around seven "bare" AGN using X-ray reflection spectroscopy. arXiv 2019, arXiv:1901.03064.

59. Zajaček, M.; Tursunov, A.; Eckart, A.; Britzen, S. On the charge of the Galactic centre black hole. Mnras 2018, 480, 4408-4423. [CrossRef]

60. Misner, C.W.; Thorne, K.S.; Wheeler, J.A. Gravitation; W. H. Freeman: San Francisco, CA, USA, 1973.

61. Wald, R.M. Black hole in a uniform magnetic field. Phys. Rev. D 1974, 10, 1680-1685. [CrossRef]

62. Chen, S.; Wang, M.; Jing, J. Chaotic motion of particles in the accelerating and rotating black holes spacetime. J. High Energy Phys. 2016, 2016, 82. [CrossRef]

63. Hashimoto, K.; Tanahashi, N. Universality in chaos of particle motion near black hole horizon. Phys. Rev. D 2017, 95, 024007. [CrossRef]

64. Dalui, S.; Majhi, B.R.; Mishra, P. Presence of horizon makes particle motion chaotic. Phys. Lett. B 2019, 788, 486-493. [CrossRef]

65. Han, W. Chaos and dynamics of spinning particles in Kerr spacetime. Gen. Relativ. Gravit. 2008, 40, 1831-1847. [CrossRef]

66. de Moura, A.P.S.; Letelier, P.S. Chaos and fractals in geodesic motions around a nonrotating black hole with halos. Phys. Rev. E 2000, 61, 6506-6516. [CrossRef] [PubMed]

67. Morozova, V.S.; Rezzolla, L.; Ahmedov, B.J. Nonsingular electrodynamics of a rotating black hole moving in an asymptotically uniform magnetic test field. Phys. Rev. D 2014, 89, 104030. [CrossRef]

68. Jawad, A.; Ali, F.; Jamil, M.; Debnath, U. Dynamics of Particles Around a Regular Black Hole with Nonlinear Electrodynamics. Commun. Theor. Phys. 2016, 66, 509. [CrossRef]

69. Hussain, S.; Jamil, M. Timelike geodesics of a modified gravity black hole immersed in an axially symmetric magnetic field. Phys. Rev. D 2015, 92, 043008. [CrossRef]

70. Jamil, M.; Hussain, S.; Majeed, B. Dynamics of particles around a Schwarzschild-like black hole in the presence of quintessence and magnetic field. Eur. Phys. J. C 2015, 75, 24. [CrossRef]

71. Hussain, S.; Hussain, I.; Jamil, M. Dynamics of a charged particle around a slowly rotating Kerr black hole immersed in magnetic field. Eur. Phys. J. C 2014, 74, 210. [CrossRef]

72. Babar, G.Z.; Jamil, M.; Lim, Y.K. Dynamics of a charged particle around a weakly magnetized naked singularity. Int. J. Mod. Phys. D 2016, 25, 1650024. [CrossRef]

73. Bañados, M.; Silk, J.; West, S.M. Kerr Black Holes as Particle Accelerators to Arbitrarily High Energy. Phys. Rev. Lett. 2009, 103, 111102. [CrossRef]

74. Majeed, B.; Jamil, M. Dynamics and center of mass energy of colliding particles around black hole in f(R) gravity. Int. J. Mod. Phys. D 2017, 26, 1741017. [CrossRef]

75. Zakria, A.; Jamil, M. Center of mass energy of the collision for two general geodesic particles around a Kerr-Newman-Taub-NUT black hole. J. High Energy Phys. 2015, 2015, 147. [CrossRef]

76. Brevik, I.; Jamil, M. Black holes in the turbulence phase of viscous rip cosmology. Int. J. Geom. Methods Mod. Phys. 2019, 16, 1950030. [CrossRef]

77. De Laurentis, M.; Younsi, Z.; Porth, O.; Mizuno, Y.; Rezzolla, L. Test-particle dynamics in general spherically symmetric black hole spacetimes. Phys. Rev. D 2018, 97, 104024. [CrossRef]

78. Shaymatov, S.R.; Ahmedov, B.J.; Abdujabbarov, A.A. Particle acceleration near a rotating black hole in a Randall-Sundrum brane with a cosmological constant. Phys. Rev. D 2013, 88, 024016. [CrossRef]

79. Atamurotov, F.; Ahmedov, B.; Shaymatov, S. Formation of black holes through BSW effect and black hole-black hole collisions. Astrophys. Space Sci. 2013, 347, 277-281. [CrossRef]

80. Narzilloev, B.; Rayimbaev, J.; Shaymatov, S.; Abdujabbarov, A.; Ahmedov, B.; Bambi, C. Can the dynamics of test particles around charged stringy black holes mimic the spin of Kerr black holes? Phys. Rev. D 2020, 102, 044013. [CrossRef]

81. Kološ, M.; Tursunov, A.; Stuchlík, Z. Possible signature of magnetic fields related to quasi-periodic oscillation observed in microquasars. Eur. Phys. J. C 2017, 77, 860. [CrossRef]

82. Kovář, J.; Kopáček, O.; Karas, V.; Stuchlík, Z. Off-equatorial orbits in strong gravitational fields near compact objects II: Halo motion around magnetic compact stars and magnetized black holes. Class. Quantum Gravity 2010, 27, 135006. [CrossRef]

83. Kovář, J.; Slaný, P.; Cremaschini, C.; Stuchlík, Z.; Karas, V.; Trova, A. Electrically charged matter in rigid rotation around magnetized black hole. Phys. Rev. D 2014, 90, 044029. [CrossRef]

84. Aliev, A.N.; Gal'tsov, D.V. Reviews of Topical Problems: “Magnetized” black holes. Sov. Phys. Uspekhi 1989, 32, 75-92. [CrossRef]

85. Aliev, A.N.; Özdemir, N. Motion of charged particles around a rotating black hole in a magnetic field. Mon. Not. R. Astron. Soc. 2002, 336, 241-248. [CrossRef]

86. Aliev, A.N.; Galtsov, D.V.; Petukhov, V.I. Negative absorption near a magnetized black hole-Black hole masers. Astrophys. Space Sci. 1986, 124, 137-157. [CrossRef]

87. Frolov, V.P.; Krtouš, P. Charged particle in higher dimensional weakly charged rotating black hole spacetime. Phys. Rev. D 2011, 83, 024016. [CrossRef]

88. Frolov, V.P. Weakly magnetized black holes as particle accelerators. Phys. Rev. D 2012, 85, 024020. [CrossRef] 
89. Stuchlík, Z.; Schee, J.; Abdujabbarov, A. Ultra-high-energy collisions of particles in the field of near-extreme Kehagias-Sfetsos naked singularities and their appearance to distant observers. Phys. Rev. D 2014, 89, 104048. [CrossRef]

90. Shaymatov, S.; Atamurotov, F.; Ahmedov, B. Isofrequency pairing of circular orbits in Schwarzschild spacetime in the presence of magnetic field. Astrophys. Space Sci. 2014, 350, 413-419. [CrossRef]

91. Abdujabbarov, A.; Ahmedov, B. Test particle motion around a black hole in a braneworld. Phys. Rev. D 2010, 81, 044022. [CrossRef]

92. Abdujabbarov, A.; Ahmedov, B.; Hakimov, A. Particle motion around black hole in Hořava-Lifshitz gravity. Phys. Rev. D 2011, 83, 044053. [CrossRef]

93. Abdujabbarov, A.A.; Ahmedov, B.J.; Shaymatov, S.R.; Rakhmatov, A.S. Penrose process in Kerr-Taub-NUT spacetime. Astrophys. Space Sci. 2011, 334, 237-241. [CrossRef]

94. Abdujabbarov, A.A.; Ahmedov, B.J.; Kagramanova, V.G. Particle motion and electromagnetic fields of rotating compact gravitating objects with gravitomagnetic charge. Gen. Relativ. Gravit. 2008, 40, 2515-2532. [CrossRef]

95. Karas, V.; Kovar, J.; Kopacek, O.; Kojima, Y.; Slany, P.; Stuchlik, Z. Regular and Chaotic Motion in General Relativity. Case of Magnetized Black Hole and a Massive Magnetic Dipole; American Astronomical Society Meeting Abstracts \#220, Anchorage, Alaska; American Astronomical Society: Washington, DC, USA, 2012; Volume 220, p. 430.07.

96. Shaymatov, S.; Patil, M.; Ahmedov, B.; Joshi, P.S. Destroying a near-extremal Kerr black hole with a charged particle: Can a test magnetic field serve as a cosmic censor? Phys. Rev. D 2015, 91, 064025. [CrossRef]

97. Stuchlík, Z.; Kološ, M. Acceleration of the charged particles due to chaotic scattering in the combined black hole gravitational field and asymptotically uniform magnetic field. Eur. Phys. J. C 2016, 76, 32. [CrossRef]

98. Rayimbaev, J.; Turimov, B.; Marcos, F.; Palvanov, S.; Rakhmatov, A. Particle acceleration and electromagnetic field of deformed neutron stars. Mod. Phys. Lett. A 2020, 35, 2050056. [CrossRef]

99. Turimov, B.; Ahmedov, B.; Abdujabbarov, A.; Bambi, C. Electromagnetic fields of slowly rotating magnetized compact stars in conformal gravity. Phys. Rev. D 2018, 97, 124005. [CrossRef]

100. Turimov, B.V.; Ahmedov, B.J.; Hakimov, A.A. Stationary electromagnetic fields of slowly rotating relativistic magnetized star in the braneworld. Phys. Rev. D 2017, 96, 104001. [CrossRef]

101. Hakimov, A.; Abdujabbarov, A.; Narzilloev, B. Quantum interference effects in conformal Weyl gravity. Int. J. Mod. Phys. A 2017, 32, 1750116. [CrossRef]

102. Shaymatov, S.; Vrba, J.; Malafarina, D.; Ahmedov, B.; Stuchlík, Z. Charged particle and epicyclic motions around 4 D EinsteinGauss-Bonnet black hole immersed in an external magnetic field. Phys. Dark Universe 2020, 30, 100648. [CrossRef]

103. Shaymatov, S. Magnetized Reissner-Nordström black hole restores cosmic censorship conjecture. Int. J. Mod. Phys. Conf. Ser. 2019, 49, 1960020. [CrossRef]

104. Rayimbaev, J.; Turimov, B.; Ahmedov, B. Braneworld effects in plasma magnetosphere of a slowly rotating magnetized neutron star. Int. J. Mod. Phys. D 2019, 28, 1950128. [CrossRef]

105. Rayimbaev, J.; Tadjimuratov, P. Can modified gravity silence radio-loud pulsars? Phys. Rev. D 2020, 102, 024019. [CrossRef]

106. Rayimbaev, J.; Turimov, B.; Palvanov, S. Plasma magnetosphere of slowly rotating magnetized neutron star in branewold. Int. J. Mod. Phys. Conf. Ser. 2019, 49, 1960019. [CrossRef]

107. Shaymatov, S.; Malafarina, D.; Ahmedov, B. Effect of perfect fluid dark matter on particle motion around a static black hole immersed in an external magnetic field. arXiv 2020, arXiv:2004.06811.

108. Narzilloev, B.; Rayimbaev, J.; Abdujabbarov, A.; Bambi, C. Charged particle motion around non-singular black holes in conformal gravity in the presence of external magnetic field. Eur. Phys. J. C 2020, 80, 1074. [CrossRef]

109. Narzilloev, B.; Rayimbaev, J.; Shaymatov, S.; Abdujabbarov, A.; Ahmedov, B.; Bambi, C. Dynamics of test particles around a Bardeen black hole surrounded by perfect fluid dark matter. Phys. Rev. D 2020, 102, 104062. [CrossRef]

110. Narzilloev, B.; Abdujabbarov, A.; Bambi, C.; Ahmedov, B. Charged particle motion around a quasi-Kerr compact object immersed in an external magnetic field. Phys. Rev. D 2019, 99, 104009. [CrossRef]

111. Shaymatov, S.; Narzilloev, B.; Abdujabbarov, A.; Bambi, C. Charged particle motion around a magnetized Reissner-Nordström black hole. Phys. Rev. D 2021, 103, 124066. [CrossRef]

112. Narzilloev, B.; Rayimbaev, J.; Abdujabbarov, A.; Ahmedov, B.; Bambi, C. Dynamics of charged particles and magnetic dipoles around magnetized quasi-Schwarzschild black holes. Eur. Phys. J. C 2021, 81, 269. [CrossRef]

113. Pradhan, P.; Majumdar, P. Circular orbits in extremal Reissner-Nordstrom spacetime. Phys. Lett. A 2011, 375, 474-479. [CrossRef]

114. Pradhan, P. Circular Geodesics, Paczyński-Witta Potential and QNMs in the Eikonal Limit for Ayón-Beato-García Black Hole. Universe 2018, 4, 55. [CrossRef]

115. de Felice, F.; Sorge, F. Magnetized orbits around a Schwarzschild black hole. Class. Quantum Gravity 2003, 20, 469-481. [CrossRef]

116. de Felice, F.; Sorge, F.; Zilio, S. Magnetized orbits around a Kerr black hole. Class. Quantum Gravity 2004, 21, 961-973. [CrossRef]

117. Rayimbaev, J.R. Magnetized particle motion around non-Schwarzschild black hole immersed in an external uniform magnetic field. Astrophys. Space Sci. 2016, 361, 288. [CrossRef]

118. Oteev, T.; Abdujabbarov, A.; Stuchlík, Z.; Ahmedov, B. Energy extraction and particle acceleration around a rotating black hole in quintessence. Astrophys. Space Sci. 2016, 361, 269. [CrossRef]

119. Toshmatov, B.; Abdujabbarov, A.; Ahmedov, B.; Stuchlík, Z. Motion and high energy collision of magnetized particles around a Hořava-Lifshitz black hole. Astrophys. Space Sci. 2015, 360, 19. [CrossRef] 
120. Abdujabbarov, A.; Ahmedov, B.; Rahimov, O.; Salikhbaev, U. Magnetized particle motion and acceleration around a Schwarzschild black hole in a magnetic field. Phys. Scr. 2014, 89, 084008. [CrossRef]

121. Abdujabbarov, A.; Rayimbaev, J.; Atamurotov, F.; Ahmedov, B. Magnetized Particle Motion in $\gamma$-Spacetime in a Magnetic Field. Galaxies 2020, 8, 76. [CrossRef]

122. Rahimov, O.G.; Abdujabbarov, A.A.; Ahmedov, B.J. Magnetized particle capture cross section for braneworld black hole. Astrophys. Space Sci. 2011, 335, 499-504. [CrossRef]

123. Rahimov, O.G. Magnetized Particle Motion around Black Hole in Braneworld. Mod. Phys. Lett. A 2011, 26, 399-408. [CrossRef]

124. Haydarov, K.; Abdujabbarov, A.; Rayimbaev, J.; Ahmedov, B. Magnetized Particle Motion around Black Holes in Conformal Gravity: Can Magnetic Interaction Mimic Spin of Black Holes? Universe 2020, 6, 44. [CrossRef]

125. Haydarov, K.; Rayimbaev, J.; Abdujabbarov, A.; Palvanov, S.; Begmatova, D. Magnetized particle motion around magnetized Schwarzschild-MOG black hole. Eur. Phys. J. C 2020, 80, 399. [CrossRef]

126. Rayimbaev, J.; Abdujabbarov, A.; Jamil, M.; Ahmedov, B.; Han, W.B. Dynamics of test particles around renormalization group improved Schwarzschild black holes. Phys. Rev. D 2020, 102, 084016. [CrossRef]

127. Vrba, J.; Abdujabbarov, A.; Kološ, M.; Ahmedov, B.; Stuchlík, Z.; Rayimbaev, J. Charged and magnetized particles motion in the field of generic singular black holes governed by general relativity coupled to nonlinear electrodynamics. Phys. Rev. D 2020, 101, 124039. [CrossRef]

128. Abdujabbarov, A.; Rayimbaev, J.; Turimov, B.; Atamurotov, F. Dynamics of magnetized particles around 4-D Einstein GaussBonnet black hole. Phys. Dark Universe 2020, 30, 100715. [CrossRef]

129. Juraeva, N.; Rayimbaev, J.; Abdujabbarov, A.; Ahmedov, B.; Palvanov, S. Distinguishing magnetically and electrically charged Reissner-Nordström black holes by magnetized particle motion. Eur. Phys. J. C 2021, 81, 124078. [CrossRef]

130. Bokhari, A.H.; Rayimbaev, J.; Ahmedov, B. Test particles dynamics around deformed Reissner-Nordström black hole. Phys. Rev. D 2020, 102, 124078. [CrossRef]

131. Dehghani, M. Thermodynamics of new black hole solutions in the Einstein-Maxwell-dilaton gravity. Int. J. Mod. Phys. D 2018, 27, 1850073. [CrossRef]

132. Dehghani, M. Thermodynamic properties of dilaton black holes with nonlinear electrodynamics. Phys. Rev. D 2018, 98, 044008. [CrossRef]

133. Dehghani, M. Thermodynamics of novel dilatonic BTZ black holes coupled to Born-Infeld electrodynamics. Phys. Rev. D 2019, 99, 024001. [CrossRef]

134. Dehghani, M. Thermodynamic properties of novel black hole solutions in the Einstein-Born-Infeld-dilaton gravity theory. Eur. Phys. J. C 2020, 80, 996. [CrossRef]

135. Rizwan, C.A.; Naveena Kumara, A.; Hegde, K.; Vaid, D. Coexistent Physics and Microstructure of the Regular Bardeen Black Hole in Anti-de Sitter Spacetime. arXiv 2020, arXiv:2008.06472.

136. Pradhan, P. Regular Black Holes as Particle Accelerators. arXiv 2014, arXiv:1402.2748.

137. Mori, K.; Gotthelf, E.V.; Zhang, S.; An, H.; Baganoff, F.K.; Barrière, N.M.; Beloborodov, A.M.; Boggs, S.E.; Christensen, F.E.; Craig, W.W.; et al. NuSTAR Discovery of a 3.76 s Transient Magnetar Near Sagittarius A*. Astron. J. Lett. 2013, 770, L23. [CrossRef]

138. Bardeen, J.M.; Press, W.H.; Teukolsky, S.A. Rotating Black Holes: Locally Nonrotating Frames, Energy Extraction, and Scalar Synchrotron Radiation. Astrophys. J. 1972, 178, 347-370. [CrossRef]

139. Novikov, I.D.; Thorne, K.S. Astrophysics of black holes. In Black Holes (Les Astres Occlus); Dewitt, C., Dewitt, B.S., Eds.; Gordon \& Breach: New York, NY, USA, 1973, pp. 343-450. 